\title{
Human monocyte-derived cells with individual hepatocyte characteristics: a novel tool for personalized in vitro studies
}

\author{
Andreas Benesic, Nora L Rahm, Samuel Ernst and Alexander L Gerbes
}

Gender, ethnicity and individual differences in hepatic metabolism have major impact on individual drug response, adverse events and attrition rate during drug development. Therefore, there is an urgent need for reliable test systems based on human cells. Yet, the use of primary human hepatocytes (PHHs) is restricted by limited availability, invasive preparation and short-term stability in culture. All other cellular approaches proposed so far have major disadvantages. We investigated whether peripheral human monocytes after cultivation according to our novel protocol (monocyte-derived hepatocyte-like cells (MH cells)) can serve as an in vitro model for hepatocyte metabolism. Enzyme activities, synthesis parameters (coagulation factor VII and urea) and cytochrome (CY) P450 activities and induction were investigated. Furthermore, $\mathrm{MH}$ cells were compared with $\mathrm{PHH}$ from the same donor. Using our protocol, we could generate cells that exhibit hepatocyte-like properties: These cells show $71 \pm 9 \%$ of specific ALT activity, $41 \pm 3 \%$ of CYP3A4 activity and $65 \pm 13 \%$ of factor VII secretion when compared with PHHs. Consequently, CYP-mediated acetaminophen toxicity and drug interactions could be shown. Moreover, the investigated parameters were stable in culture over at least 4 weeks. Furthermore, MH cells retain gender-specific and donor-specific CYP activities and toxicity profiles, respectively. $\mathrm{MH}$ cells show quantitative and qualitative approximation to human hepatocytes concerning CYP-metabolism and toxicity. Our data support individual prediction of toxicity and CYP metabolism. MH cells are a novel tool to investigate long-term hepatic toxicity, metabolism and drug interactions.

Laboratory Investigation (2012) 92, 926-936; doi:10.1038/labinvest.2012.64; published online 2 April 2012

KEYWORDS: cytochrome P450; hepatocyte model; in vitro testing; personalized medicine; toxicity

The liver is an important site of drug metabolism and hepatocytes are mainly responsible for toxification and detoxification of substances. A huge array of enzymes and transporters allows the hepatocyte to metabolize, conjugate and finally excrete xenobiotics and endobiotics. The first step, also referred to as phase-I metabolism, is performed predominantly by enzymes of the cytochrome P450 (CYP450) enzyme family. The CYPs 1A2, 2A6, 2B6, 2D6, 2E1, 3A4 and $3 \mathrm{~A} 5$ are found in hepatocytes with great abundance. ${ }^{1}$ Most CYP enzymes can be induced, that is, their activity in the hepatocyte is increased to manage greater amounts of xenobiotics. This induction is mediated by receptors, such as the pregnane-X-receptor (PXR), ${ }^{2}$ the aryl-hydrocarbon receptor ${ }^{3}$ and the constitutive androestendione receptor. ${ }^{1}$ Knowing the way a substance is metabolized and how a substance interacts with co-medication is crucial for modern drug design. Therefore, hepatocyte models are urgently needed for drug development, because no other cell possesses a comparable equipment of metabolism pathways. The complexity of this system is augmented by different activities of CYP enzymes dependent on sex, age, ethnicity and individual factors, including co-medication. ${ }^{4-7}$ Pharmacogenomics is a powerful tool to identify different individual metabolism patterns, ${ }^{8}$ yet this method only detects genetic polymorphisms, which not always predict the actual function of the enzyme., ${ }^{9,10}$ On the search for a valid in vitro hepatocyte model, several different cell types have been investigated.

Obviously, the use of primary hepatocytes as an in vitro model should be the golden standard for metabolism and toxicity studies in vitro. On the first glance, primary hepatocytes 
possess all the required activities and short-term studies can be performed with valid results. ${ }^{11,12}$ Yet, primary hepatocytes dedifferentiate in culture dependent on the used culture conditions (plated monolayer, sandwich culture and suspension culture). This dedifferentiation process results in a loss in CYP450 activities and inducibility within few days. ${ }^{13}$ Using co-cultures and 3D-models, dedifferentiation can be slowed, yet these improvements do not enable experiments over 28 days as required by regulatory authorities. This precludes the use of primary hepatocytes for long-term experiments. Another drawback of primary hepatocytes is their low abundance and the invasive way of preparation (liver biopsy, partial liver resection, hepatocytes from cadaveric livers). Ethical concerns preclude the gain of primary human hepatocytes (PHHs) in many countries. On this basis, human hepatocytes can be acquired in a sufficient amount for in vitro experiments, but because of the mentioned restrictions, it is very difficult to investigate gender, ethnic or individual differences using primary hepatocytes. In order to screen a substance, the use of hepatocytes from animals can be an alternative, but species differences in dose-effect relation can be of significant relevance.

In order to overcome the problems with primary hepatocytes, different technologies using stem cells were developed in the recent years. Promising results with respect to hepatocyte characteristics could be obtained using the differentiation of embryonic stem cells (ES cells) into hepatocytes. However, the ethics of using ES cells are controversially discussed and there are major restrictions on ES cell research in many countries. There is evidence for bone marrow stem cells, mesenchymal stem cells or inducible pluripotent stem cells to be a potential in vitro or even in vivo replacement for primary hepatocytes. Yet, the generation of these cells takes about 4-6 weeks, the yield is rather low and functional hepatocyte characteristics are inconsistent. None of these models has been established as an alternative for hepatocytes in vitro and because of the low yields and long generation times the process of validation will be cumbersome.

Another way to use cells with high yields and hepatocytecharacteristics is the use of immortalized hepatoma cells, which retain hepatocyte function despite malignant dedifferentiation. Cell lines such as HepG2, Hep3B and Huh-7 are well established, but they lack several hepatocyte functions. HepG2 cells, for example, exhibit functional CYP450 enzymes, yet their function strongly depends on source and culture conditions ${ }^{14}$ and they lack a functional urea-cycle. ${ }^{15}$ Other hepatoma cell lines that have been proposed as better models for in vitro hepatocyte research include Fa2N-4, HC04 and HepaRG cells, which show active and inducible CYP450 metabolism and similar toxicity profiles as human hepatocytes. An important drawback of these tumor cell models is the origin from only one donor and the difficulty to predict stable functions. ${ }^{14}$

A very innovative way to generate hepatocyte-like mouse cells was shown in the work of Sekiya and Suzukai ${ }^{16}$ as well as
Huang et al. ${ }^{17}$ These groups could show that the genetic induction of special combinations of transcription factors can convert mouse fibroblasts to hepatocyte-like phenotype.

In the last years, there were reports that human peripheral blood mononuclear cells or monocytes can acquire hepatocyte characteristics without genetic modifications under certain conditions. For example, it has been shown that peripheral monocytes from hepatitis B-infected individuals can engraft into mouse liver and express different hepatocyte typic molecules, such as albumin. ${ }^{18}$ Another work showed that monocytes are capable to attenuate liver damage by acetaminophen (APAP) in a mouse model. ${ }^{19}$ Plasticity of monocytic cells and the possibility to transdifferentiate across lineage barriers, as shown for lymphocytes, ${ }^{20}$ has been proposed. Prior work showed first evidence for acquisition of hepatocyte-like properties in monocytes, ${ }^{21}$ yet the protocol was afflicted with problems. ${ }^{22}$ The use of autologous donor sera seems to alleviate the standardization problems with this model to some degree, yet the use of serum makes cells susceptible for variations caused by different batches of serum. $^{23}$

Thus, the generation of cells with hepatocyte characteristics from monocytes could be a promising alternative solution of the problems, which the current in vitro models are afflicted with: short-lived enzyme activities, missing possibilities for individualization, difficult or cumbersome generation and low abundance of material.

We here present the features of our in vitro hepatocyte model from human peripheral monocytes.

\section{MATERIALS AND METHODS Cell Culture}

Monocyte isolation and culture

Human peripheral monocytes were acquired from healthy donors after informed consent. Blood was collected and cell processing started immediately. Monocytes were isolated after centrifugation of whole blood over a Ficoll gradient. Afterward the interphase was collected, washed three times with ice-cold phosphate-buffered saline (PBS) and cells were plated in the appropriate culture vessels. Cells were cultured at $37^{\circ} \mathrm{C}$ and $5 \% \mathrm{CO}_{2}$ in a standard incubator. Serum-free incubation medium was composed according to the standard operating procedure reported in our patent application (EPA \#10172639.6 and PTC/EP2011/063871). Cells were cultured in medium 1 (DMEM/HAM-F12 containing 2\% human albumin, $100 \mathrm{IE} / 100 \mu \mathrm{g}$ penicillin/streptomycin, $1 \mathrm{ng} / \mathrm{ml}$ recombinant human interleukin-3, $0.5 \mathrm{ng} / \mathrm{ml}$ recombinant human macrophage colony-stimulating factor, $2 \mathrm{mM}$ L-glutamine, $100 \mathrm{nM}$ adenosine, $10 \mu \mathrm{M}$ deoxycholate and $50 \mu \mathrm{M}$ caffeine) for 5 days and in medium 2 (DMEM/HAM-F12 containing $2 \%$ human albumin, $100 \mathrm{IE} / 100 \mu \mathrm{g}$ penicillin/ streptomycin, $3 \mathrm{ng} / \mathrm{ml}$ recombinant human fibroblast growth factor- $4,2 \mathrm{mM}$ L-glutamine, $5 \mathrm{IE} / \mathrm{ml}$ unfractionated heparin, $0.28 \mathrm{mU} / \mathrm{ml}$ recombinant human insulin, $5 \mathrm{nmol} / \mathrm{l}$ recombinant human glucagon, $10 \mathrm{ng} / \mathrm{ml}$ recombinant human epidermal 
growth factor, $10 \mu \mathrm{M}$ deoxycholate and $10 \mu \mathrm{M}$ caffeine) for the following culture period up to a total of 42 days (from isolation). Experiments were conducted at the end of the generation process at day 10 or later after isolation. Typically, cells were used for experiments 14 days after isolation (day 0 ) up to 28 days in culture.

Cryoconservation was performed using DMEM/HAM-F12 medium containing $20 \%$ DMSO, L-glutamine $2 \mathrm{mM}$, penicillin/streptomycin and $2 \%$ human serum albumin and sterile PBS containing 1\% human serum albumin in a $1: 1$ mixture. Cells were stored at $-80^{\circ} \mathrm{C}$ for 14 days.

Patient monocytes were obtained according to the guidelines of the charitable state controlled foundation HTCR (Human Tissue and Cell Research, www.htcr.de; see also next paragraph).

\section{PHH isolation and culture}

PHHs were obtained from the foundation human tissue and cell research (www.htcr.de). Tissue samples and annotated data were obtained and experimental procedures were performed within the framework of the non-profit foundation HTCR, including the informed patient's consent. ${ }^{24}$ Three patients donated blood samples for monocyte preparation before partial liver resection. Hepatocytes were cultured as described elsewhere ${ }^{25}$ and were used for experiments at day 4 after isolation. Before stimulation or induction experiments, cells were starved in medium without additives to exclude possible interactions of medium components and chemicals.

\section{Chemicals and Reagents}

Gly-L-pro-p-nitroanilide, L-gamma-glutamyl-p-nitroanilide and glycylglycine to gamma-glutamyl-glycylglycide and p-nitroaniline, rifampicin, carbamazepine, artemisine and 6-(4-chlorophenyl)imidazo[2,1-b][1,3]-thiazole-5-carbaldehyde O-(3,4-dichlorobenzyl)oxime (CITCO) were purchased by Sigma-Aldrich, Deisenhofen Germany. All other chemicals used were of analytical grade and provided by Merck, Darmstadt, Germany.

\section{FACS-Analysis}

Expression of leukocyte and monocyte markers was determined by FACS analysis. Detailed description is provided in the Supplementary Material.

\section{Enzyme Activities}

Alanine aminotransferase (ALT)

Specific ALT activity was determined spectrophotometrically as described by Schumann et al. ${ }^{26}$

\section{Dipeptidylpeptidase (DPP) IV}

Specific activity of DPP IV was determined spectrophotometrically (see Supplementary Material).
Gamma-glutamyl-transpeptidase (gamma-GT)

Gamma-GT activity was measured using a standard proto$\mathrm{col}^{27}$ For details please refer to Supplementary Material.

\section{Lactate dehydrogenase (LDH)}

LDH activity was determined spectrophotometrically by a standard protocol described in the Supplementary Material.

\section{Urea Synthesis}

Secretion of urea in the cell culture supernatant was determined using QuantiChromTM Urea Assay Kit (BioAssay Systems, Hayward, CA, USA) according to the manufacturer's instructions.

\section{Toxicity Experiments}

Toxicity was determined by release of LDH measured using the CytoTox 96 Non-Radioactive Cytotoxicity Assay (Promega, Madison, WI, USA) according to the manufacturer's instructions. LDH-release was calculated as percentage of $\mathrm{LDH}$ released in the culture media of total $\mathrm{LDH}$.

\section{Measurement of Non-Protein Thiol Groups}

Non-protein thiols as a surrogate marker for oxidative stress were measured using the reagent of Ellman (5,5'-dithio-bis2-nitrobenzoic acid) as described elsewhere. ${ }^{24}$

\section{Cytochrome (CYP) P450 Activities and Induction}

Activities of the CYP450 enzymes 3A4, 2C9 and 1A2 were determined using P450-GloTM Assay (Promega) according to the manufacturer's instructions. Briefly, cells were induced for various periods with known CYP-inducers in media without additives. ${ }^{28}$ Luminescence values were normalized for protein content.

\section{Factor VII-ELISA}

For analysis of secreted factor VII, a direct ELISA was performed as described in Supplementary Material.

\section{Western Blotting}

Protein expression of CYP2E1, 2C9 and 3A4 in cell lysates and factor VII in supernatants was determined by western blotting. Detailed information is provided in the Supplementary Files.

\section{c-RNA Microarrays}

To screen expression pattern for genes involved in metabolism and toxicity, a c-RNA Microarray platform from SA Biosciences (Frederick, MD, USA) was used (Oligo GEArray Toxicology and Drug Resistance Microarray Catalogue \#OHS-401). The experimental design and analysis strategy as well as all data sets are provided in the supplements. Briefly, total RNA was isolated from monocyte-derived hepatocytelike cells (MH cells) 14 days after isolation and from hepatocytes 4 days after isolation, using the total RNA Isolation Kit (SA Biosciences) according to the manufacturer's 
instruction. After synthesis of biotinylated c-RNA and hybridization according to the manufacturer's instructions, expression levels of genes were analyzed by X-ray detection and analysis of array signal using TIGR spotfinder software. Gene expression was expressed as $\log 2$ normalized to betaactin.

\section{Statistics}

The data are presented as mean values \pm s.d. Significance of differences was tested by the paired or unpaired Student's $t$-test or ANOVA as appropriate (SPSS Software version 15.0). Differences were considered significant at $P<0.05$. $N$ represents the number of donors. Measurements from cells of a single donor were performed as triplicates or quadruplicates.

\section{RESULTS}

\section{Cell Characteristics and Yield}

During culture of monocytes using our protocol there is a marked increase in cell size and at day 14 cells show an epitheloid growth pattern (microscope images are provided in the Supplementary Files). FACS analysis after the isolation process reveals $64 \pm 15 \%$ CD14-positive cells with a small percentage (CD3-positive cells $8 \pm 6 \%$ ) of contaminating lymphocytes. In all, $88 \pm 8 \%$ of the cells were CD 45 -positive ( $n=10$ donors). During culture, there is a moderate increase in cell number $(2.3 \pm 0.3$-fold; $P<0.05, n=4)$, this is paralleled by a marked increase in cellular protein $(8.8 \pm 1.7$-fold, $P<0.05, n=12)$ and RNA-content $(4.4 \pm 1.7$-fold, $P<0.05$, $n=3$ ) most probably due to hypertrophy. Using FACS analysis, cells at day 14 stained positive for CYP2E1 and 3A4 (data not shown).

At day 14, cells show activities of various hepatocyte characteristic enzymes such as DPP IV, LDH, gamma-GT and the liver-specific ALT. Compared with PHHs these enzymes reach specific activities of $78 \pm 19 \%$ (DPP IV), $94 \pm 11 \%$ (gamma-GT), $71 \pm 12 \%$ (ALT) and $73 \pm 28 \%$ (LDH) in cells of monocytic origin ( $n=8$ for MH cells and $n=3$ for $\mathrm{PHH}$, see also Supplementary Material).

\section{MH Cells Display CYP450 Activity and Induction}

In order to measure CYP450 activities in MH cells, we used luminescence based assays for CYP450 1A2, 2C9 and 3A4. ${ }^{28}$

$\mathrm{MH}$ cells exhibit activities of the CYP450 enzymes 3A4, 2C9 and 1A2. CYP3A4 shows highest luminescence values, followed by 1A2 and 2C9 (Figure 1a). This activity pattern is comparable to the situation in the liver.

To investigate induction of CYP enzymes cells were incubated with the inducers rifampicin and 3-methylcholanthrene (3MC). This led to a significant increase in CYP activities (Figure 1a). Protein content, as a crude marker for cell viability was not reduced by treatment with rifampicin (124 $\pm 12 \%$ of control) or $3 \mathrm{MC}(118 \pm 15 \%$ of control).

As CYP-induction is dose dependent, we tested different concentrations of the CYP 1A2-inducer 3MC on CYP1A2 and rifampicin on CYP3A4 activity. The results shown in Figures $1 \mathrm{~b}$ and $\mathrm{c}$ provide evidence for a dose-dependent CYPinduction in $\mathrm{MH}$ cells.

To test CYP-induction via different pathways rifampicin, phenytoin, carbamazepin, artemisin ${ }^{1}$ and 6-(4-chlorophenyl)imidazo[2,1-b] [1,3]-thiazole-5-carbaldehyde O-(3,4-dichlorobenzyl)oxime (CITCO) ${ }^{29}$ and dexamethasone ${ }^{1}$ were used. The results are shown in Figure 1d: all tested substances affected an increase in CYP3A4 activity. None of these substances caused relevant toxicity during the 48-h incubation period or had significant influence on protein content (phenytoin $5 \mu \mathrm{M}$ : $102 \pm 21 \%$; phenytoin: $91 \pm 8 \%$, carbamazepin: $99 \pm 22 \%$, CITCO: $111 \pm 9 \%$, artemisin: $88 \pm 25 \%$ and dexamethasone: $86 \pm 8 \%$ of control).

In order to support the results obtained with CYP function, CYP enzymes and their induction were investigated by western blotting using specific antibodies for CYP 2E1, 3A4 and $2 \mathrm{C} 9$. The presence of these enzymes is confirmed by specific western blot bands. Furthermore, there is also an increase in CYP-protein after pre-treatment of the cells with inducers (ethanol for $2 \mathrm{E} 1$, rifampicin for $2 \mathrm{C} 9$ and $3 \mathrm{~A} 4$; Figure 1e).

\section{MH Cells are Susceptible to Hepatotoxic Drugs and Drug Interactions}

As toxification of substances is a major problem in drug development, we investigated, whether $\mathrm{MH}$ cells are able to toxify APAP, an analgesic drug that is known to be converted to toxic $\mathrm{N}$-acetyl-pbenzoquinone-imine in the liver by CYP $2 \mathrm{E} 1{ }^{30}$ APAP exposure led to a time and dose-dependent increase in LDH-release (Figure 2a), suggesting cytochromemediated generation of toxic metabolites. By measurement of free thiols, we found in parallel to the mechanism described in hepatocytes a decrease in thiols in MH cell after APAP exposure (Figure 2b).

Diclofenac is a hepatotoxic analgesic that causes liver damage through direct toxicity, toxicity of metabolites and allergic reaction. ${ }^{31,32}$ Figure $2 \mathrm{c}$ shows time and dose dependency of diclofenac toxicty in $\mathrm{MH}$ cells.

As $\mathrm{MH}$ cells are susceptible to toxicity induced by APAP and diclofenac, respectively, interactions of these drugs with inducers of CYP enzymes were assessed. CYP2E1 has a role in alcohol metabolism and progression of hepatic fibrosis. ${ }^{33}$ To test CYP2E1 involvement in the toxicity of APAP in MH cells, cells were pre-treated $48 \mathrm{~h}$ with ethanol to increase CYP2E1 content. ${ }^{34}$ The results shown in Figure $2 \mathrm{~d}$ indicate that ethanol pre-treatment increases APAP toxicity. This finding is in parallel to clinically relevant aggravation of APAP-induced liver damage by ethanol consumption.

$\mathrm{N}$-acetyl-cysteine is used as an antidote for APAP poisoning and has been shown to be protective in vivo and in vitro. As in the clinical setting, $\mathrm{N}$-acetyl-cysteine co-administration prevented APAP toxicity in MH cells (Figure 2e). 
a
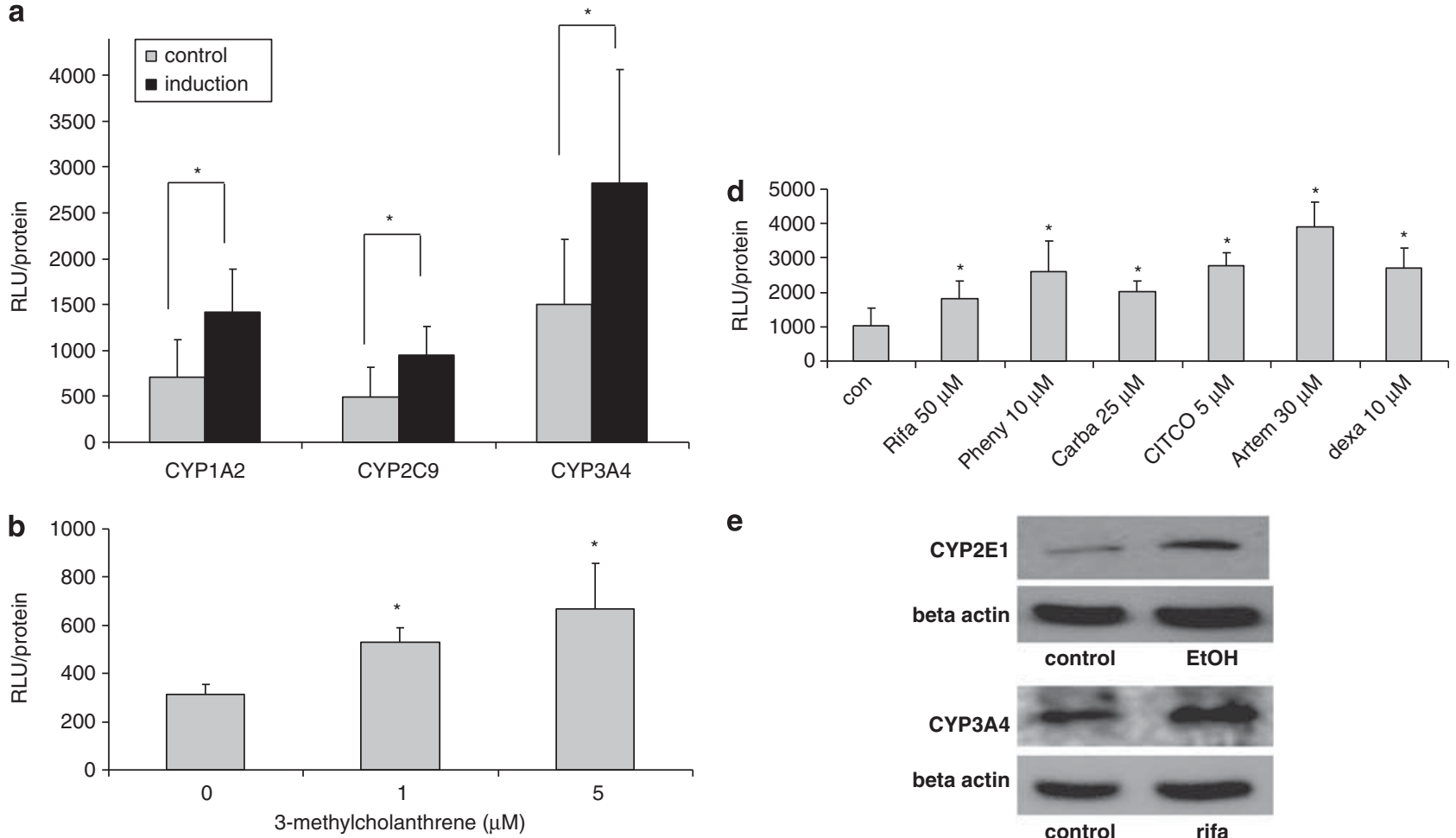

e
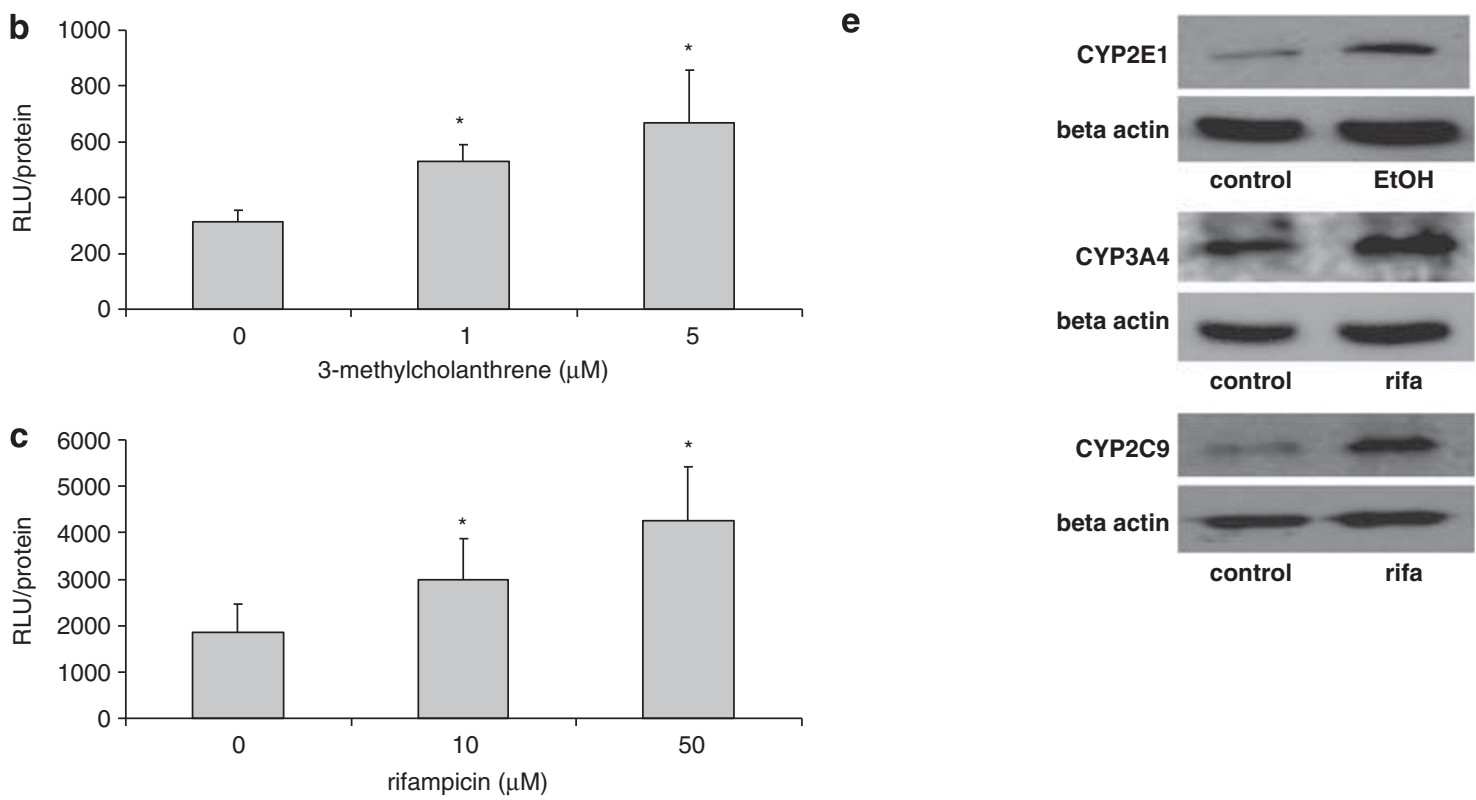

Figure 1 (a) Activities of the CYP450 enzymes 1A2, 2C9 and 3A4 measured by luminescence assay. At 48-h incubation with prototypical inducers (1A2: $5 \mu \mathrm{M}$ 3MC, 2C9 and 3A4: $50 \mu \mathrm{M}$ rifampicin) leads to an increase in CYP activities (CYP1A2, 2C9: $n=12 ;$ CYP3A4: $n=14 ;{ }^{*} P<0.05$ vs control). (b) Dose-dependent CYP1A2 induction by $48 \mathrm{~h}$ incubation with 3MC measured by CYP1A2 activity $\left(n=4,{ }^{\star} P<0.05\right.$ vs control). (c) Dose-dependent CYP3A4 induction by $48 \mathrm{~h}$ incubation with rifampicin ( $n=4,{ }^{*}<0.05$ vs control). (d) Induction of CYP3A4 activity by 48-h incubation with rifampicin and phenytoin (PXR), carbamazepine, CITCO and artemisine (constitutive androstendione receptor) and dexamethasone (glucocorticoid receptor) ( $n=4 ;{ }^{\star} P<0.05$ vs control). (e) Western blot scans for CYP2E1, 3A4 and 2C9 in lysates of hepatocyte-like cells with and without induction (CYP2E1: 2\%o ethanol for 48 h, CYP3A4 and 2C9: $50 \mu \mathrm{M}$ rifampicin for $48 \mathrm{~h}$ ). Beta-actin was used as loading control.

As metabolism of diclofenac by CYP2C9, 2C19 and 3A4 can enhance toxicity by formation of toxic metabolites, we investigated diclofenac toxicity after CYP induction using $48 \mathrm{~h}$ pre-incubation with rifampicin. CYP induction using $48 \mathrm{~h}$ pre-incubation with $100 \mu \mathrm{M}$ rifampicin led to an increase in diclofenac toxicity, depicted in Figure $2 \mathrm{f}$.

\section{MH Cells Show Hepatocyte Characteristic Synthesis}

An important function of hepatocytes is the detoxification of ammonia to urea, therefore we investigated urea secretion of $\mathrm{MH}$ cells. Basal urea secretion was increased after incubation with different ammonium chloride concentra- tions (Figure 3a). These data provide evidence for a functional ammonia detoxification by hepatocyte-like cells of monocytic origin.

Another fundamental characteristic of hepatocytes is the ability to synthesize coagulation factors. As synthesis coagulation factor VII in the liver is vitamin $\mathrm{K}$ dependent, we tested whether MH cells secrete factor VII into the supernatant and the dependence on vitamin $\mathrm{K}$. After incubation with different concentrations of menadione, specific western blot bands in cell culture supernatant and ELISA-signals revealed a menadione-dependent secretion of factor VII (Figures $3 \mathrm{~b}$ and $\mathrm{c}$ ). 

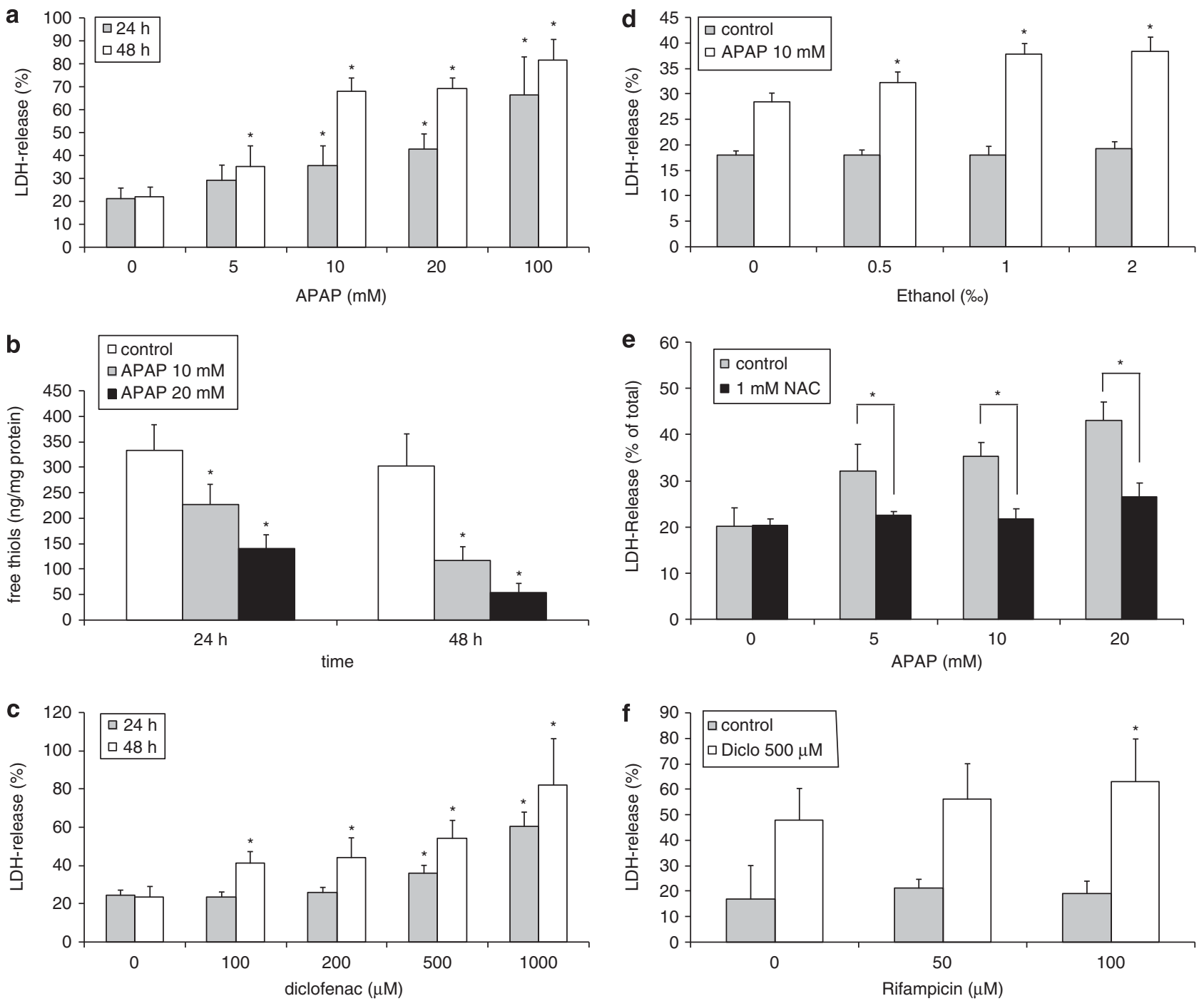

Figure 2 (a) Time- and dose-dependent effect of APAP on MH cells ( $n=6, * P<0.05$ vs control). (b) Time- and dose-dependent decrease in thiols in MH celllysate because of APAP treatment. Values were measured at $t=24$ and $48 \mathrm{~h}$, respectively, and normalized for protein content ( $n=3, * P<0.05 v s$ control). (c) Time- and dose-dependent toxicity of diclofenac $(n=4, * P<0.05$ vs control). (d) Pre-treatment with the CYP2E1-inducer ethanol for $48 \mathrm{~h}$ significantly enhances toxicity of 24-h exposure to $10 \mathrm{mM}$ APAP $(n=4, * P<0.05)$. (e) Co-administration of APAP and $N$-acetylcysteine (NAC) abolishes the toxic effect of APAP ( $n=4,{ }^{*} P<0.05$ vs control). (f) Pre-treatment with rifampicin to induce CYP 2 C9 and $3 \mathrm{~A} 4$ for $48 \mathrm{~h}$ increases the toxic effect of $1000 \mu \mathrm{M}$ diclofenac after 24-h exposure $\left(n=4,{ }^{*} P<0.05\right)$.

\section{MH Cells Exhibit Stable Hepatocyte Characteristics Over 28 Days}

To investigate superiority of the cell model presented in the article, we investigated the stability of the hepatocyte-like functions. Toxicity and CYP activities and induction were of special interest. CYP3A4 activity over time in culture, CYP induction and APAP toxicity are summarized in Table 1 . There is only a slight loss in basal activity during a culture period of 4 weeks. Furthermore, CYP inducibility seems also to be stable over 4 weeks in culture. Moreover, toxicity of APAP is stable over 28 days in culture.

\section{MH Cells Characteristics Show Approximation to PHHs}

In order to investigate hepatocyte characteristics quantitatively, enzyme activities, CYP activities and inducibility as well as synthesis of urea and factor VII were analyzed in comparison with PHHs. By western blotting, we found $47.2 \pm 4.4 \%$ of CYP3A4 content in lysates of $\mathrm{MH}$ cells compared with PHHs. CYP 2E1 was found with an even higher expression of $58.2 \pm 10 \%$ of primary hepatocytes (Supplementary Material). Analysis of cell culture supernatant revealed $54.7 \pm$ $13.6 \%$ of PHH factor VII secretion (Table $2 ; n=3$ ). Furthermore, we compared the functional characteristics of PHHs from three individual donors who underwent liver resection and donated hepatocytes. $\mathrm{MH}$ cells derived from peripheral monocytes show remarkable approximation to $\mathrm{PHH}$ from the same donor (Table 2). 

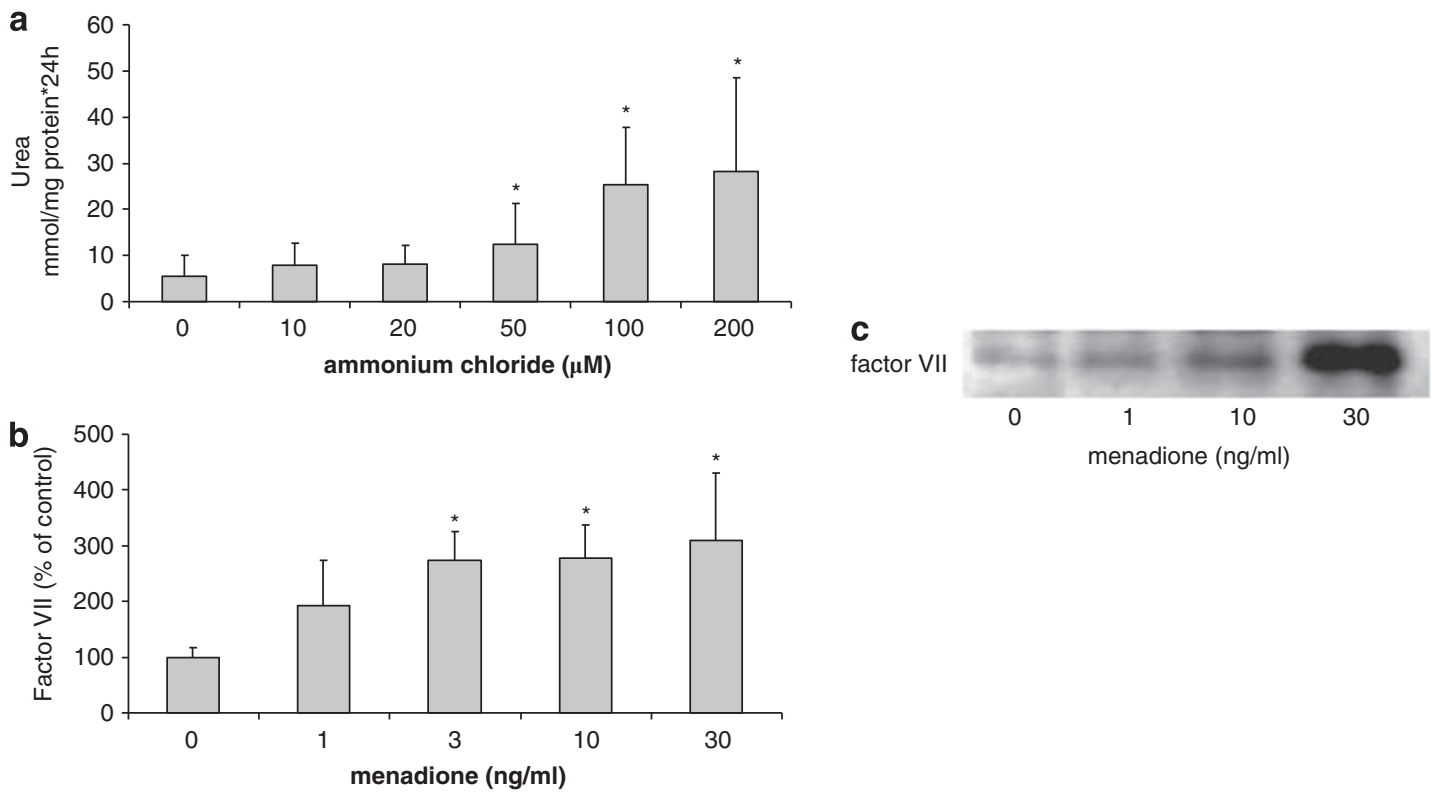

Figure 3 (a) At 24-h exposure to ammonium chloride leads to an increase in urea secretion that is significant when $>50 \mu \mathrm{mol} / \mathrm{l}$ ammonium chloride are used $\left(n=4,{ }^{\star} P<0.05\right)$. (b) MH cells secrete factor VII as determined by ELISA. This secretion can be enhanced by treatment with menadione for $24 \mathrm{~h}$ ( $n=6$, $\left.{ }^{*} P<0.05\right)$. (c) Western blot of cell culture supernatant confirms the increase in factor VII after menadione treatment.

Table 1 Time course of CYP3A4 activity, CYP3A4 induction and APAP toxicity during long-term culture of MH cells

\begin{tabular}{lccccc}
\hline CYP3A4 & Day 0 & Day 7 & Day 14 & Day 21 & Day 28 \\
\hline \% Basal activity & 100 & 88 & 104 & 83 & 64 \\
S.d. & 70 & 63 & 31 & 26 & 15 \\
P vs basal day 0 & & 0.77 & 0.65 & 0.39 & 0.25 \\
X-fold induction & 1.9 & 2.0 & 2.1 & 2.1 & 1.9 \\
S.d. & 0.9 & 0.6 & 0.6 & 0.7 & 0.7 \\
$P$ (con vs induced) & 0.046 & 0.022 & 0.022 & 0.032 & 0.003 \\
N & 7 & 5 & 4 & 4 & 3 \\
& & & & & \\
APAP toxicity & & & & & \\
Delta LDH release & 22 & 19 & 19 & 21 & 22 \\
S.d. & 6 & 8 & 5 & 7 & 5 \\
$P$ vs basal day 0 & & 0.47 & 0.38 & 0.42 & 0.32 \\
$N$ & 6 & 3 & 4 & 4 & 3 \\
\hline
\end{tabular}

There is a slight decrease in CYP3A4 activity after 28 days in culture, yet the inducibility is maintained throughout the culture period. APAP toxicity as a surrogate for CYP2E1 activity is stable during 28 days in culture.

\section{MH Cells Reflect Gender Differences in Toxicity and CYP Activity}

Gender differences in hepatic metabolism are subject of intensive investigations and their importance is underlined by the fact that women suffer twice as often from drug- induced liver injury compared with men. In order to evaluate whether monocyte-derived cells could prove useful for individualized toxicity testing, we incubated cells of female and male donors with APAP and diclofenac. As shown in Figure $4 \mathrm{a}$, there is no difference in APAP toxicity between cells derived from female and male donors. Yet, there is a significant greater toxicity of diclofenac (which is predominantly metabolized by 3A4) in cells derived from female subjects. This finding is paralleled by a significantly higher activity of $3 \mathrm{~A} 4$ in cells derived from female donors, as shown in Figure $4 \mathrm{~b}$.

\section{MH Cells can be Cryoconserved}

In order to establish a cell bank from different donors, cryoconservation of the cells is required. Comparison of fresh and cryoconserved $\mathrm{MH}$ cells revealed an increase in $\mathrm{LDH}$ release after 24 -h exposure to $20 \mathrm{mM}$ APAP of $21.6 \pm 9.0 \%$ in fresh vs $25.0 \pm 9.2 \%$ in cryoconserved $\mathrm{MH}$ cells $(n=5$, $P=0.39$ ). Moreover, 24 -h challenge with $1 \mathrm{mM}$ diclofenac induced a rise in $\mathrm{LDH}$ release to $58.2 \pm 9.8 \%$ in fresh and $50.3 \pm 9.6 \%$ in cryoconserved cells, respectively, $(n=5$; $P=0.24)$. Thus, cryopreservation of MH cells seems possible without loss of important features.

\section{MH Cells Reflect Individual Differences in CYP Activity and Expression}

As there seem to be gender differences in toxicity and CYP activities of MH cells, we investigated individual CYP activities and their inducibility in hepatocytes and cells of monocytic origin derived from the same donor. As shown in Table 3, basal activities and the induction of CYP3A4 and 
Table 2 Hepatocyte functions compared quantitatively between PHH and MH cells

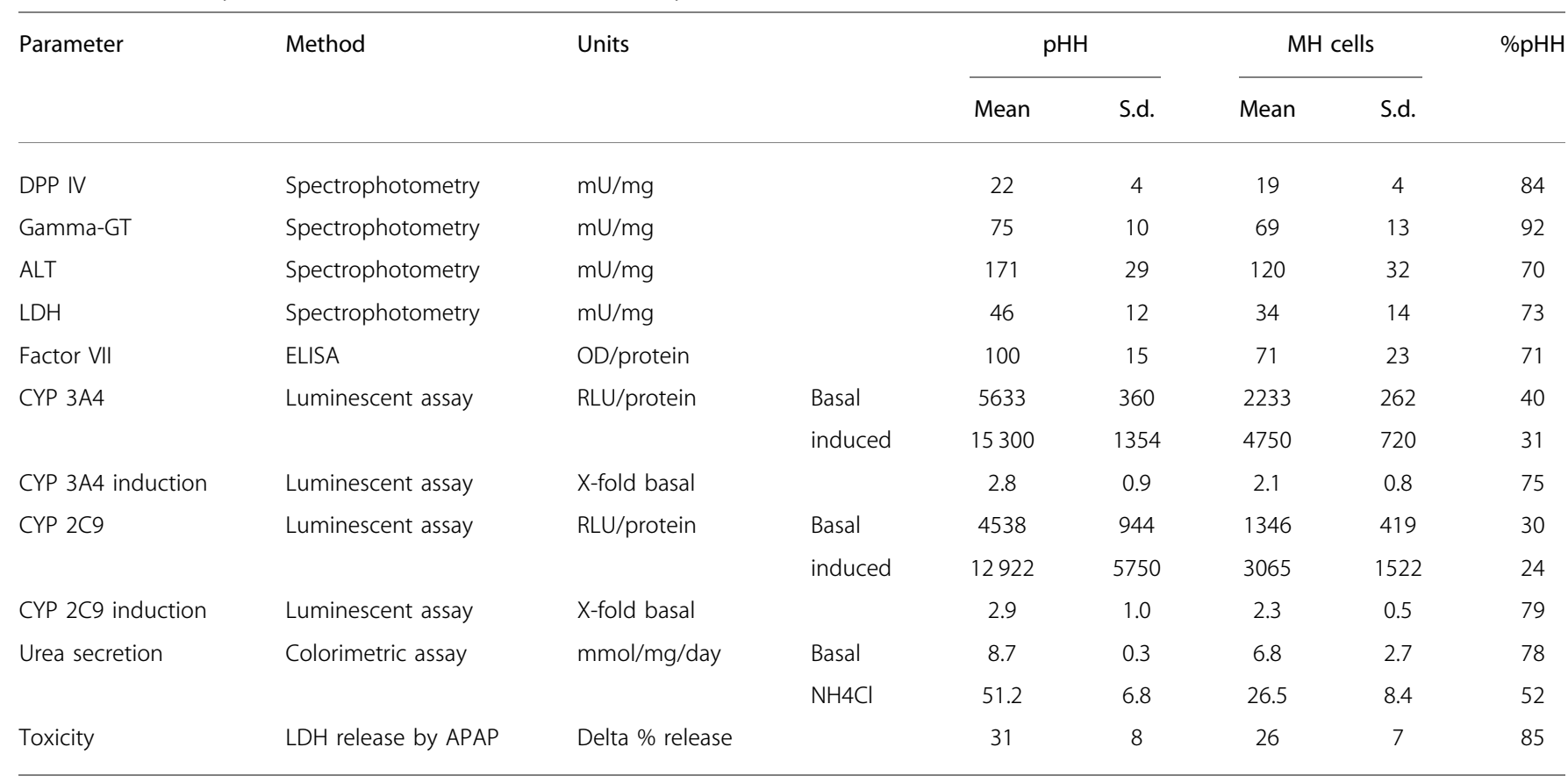

MH cells show similar specific activities pf DPP IV, ALT, gamma-GT and LDH compared with PHH derived from the same donors ( $n=3$ ). Activities of CYP3A4 and $2 \mathrm{C} 9$ in $\mathrm{MH}$ cells reach $40 \%$ and $30 \%$ of PHH, respectively. A detailed comparison of activities and inducibility in PHH and $\mathrm{MH}$ cells of the individual donors is given in Table 3.

a

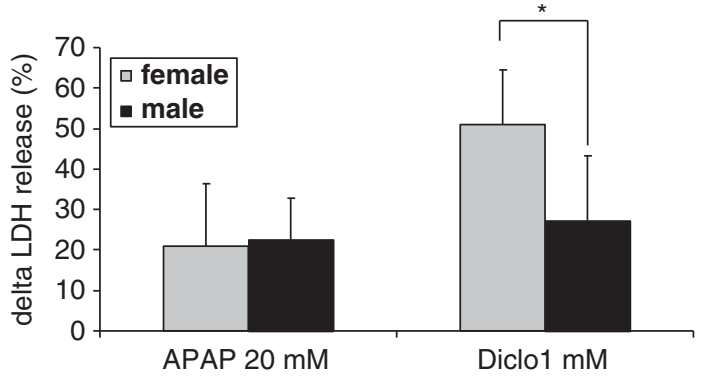

b

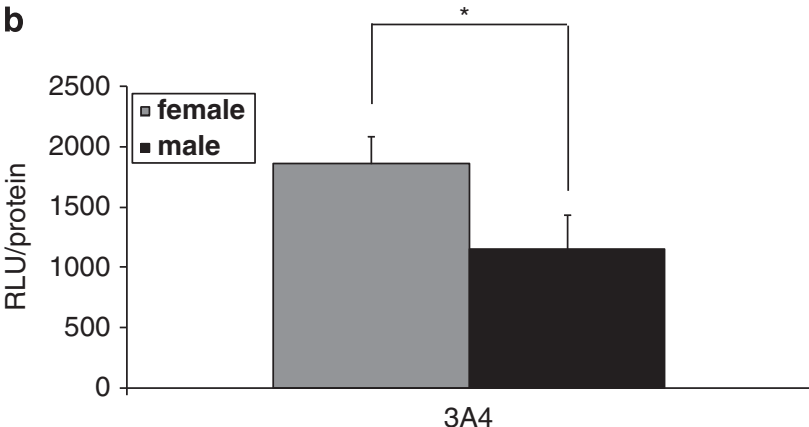

Figure 4 (a) Diclofenac, but not APAP toxicity is higher in cells from female donors ( $n=6$ female, 6 male, ${ }^{\star P}<0.05$ ). (b) CYP3A4 activity is higher in MH cells of female origin $\left(n=6\right.$ female and 6 male; $\left.{ }^{*} P<0.05\right)$.

2C9 in primary hepatocytes seem to be reflected by $\mathrm{MH}$ cells derived from the same donor.

To investigate this more in detail, we used c-RNA microarrays to analyze gene expression profiles in $\mathrm{MH}$ cells and
PHHs from the same donor. Comparing expression of transcription factors, a close correlation between $\mathrm{MH}$ cells and the corresponding $\mathrm{PHH}$ was observed in all three donors (Figure 5a). Expression of CYP-RNA was of special interest and data are shown in Figures $5 \mathrm{~b}$ and $\mathrm{c}$. The mRNA levels of important CYP450 enzymes seem to correlate between $\mathrm{MH}$ cells and PHHs, supporting the presented functional data. Thus, there is evidence for reflection of individual hepatic CYP450 equipment by MH cells.

\section{DISCUSSION}

The aim of this study was to investigate the potential usefulness of hepatocyte-like cells of peripheral monocytic origin as a tool for in vitro research. There is urgent need for a valid, stable and predictive tool for liver-specific toxification and detoxification processes as well as CYP450 metabolism. Primary hepatocytes can be of use, but these cells dedifferentiate in culture. ${ }^{12}$ Therefore, primary hepatocytes are not suited for long-term experiments. Other models have been proposed which make use of immortal human hepatoma cell lines that retain to some extent hepatocyte-specific function, especially CYP450 enzyme activities and their inducibility. Yet, tumor cell lines are derived from only one 'donor' and most probably do not reflect the situation in the population properly. Moreover, hepatoma cell lines are not cultivated under stringent conditions and therefore research data may vary between laboratories. ${ }^{13}$ To overcome these problems, stem cells hold great promise, but the generation of hepatocytes from stem cells is burdened by ethical problems as 
Table 3 Comparison of CYP3A4 and 2C9 activity (arbitrary units) under basal conditions and after induction with $50 \mu \mathrm{M}$ rifampicin in hepatocytes (pHH) and MH cells of three different donors

\begin{tabular}{ccccc}
\multicolumn{2}{c}{$\mathrm{PHH}$} & \multicolumn{2}{c}{$\mathrm{MH}$} \\
\cline { 5 - 6 } & Basal Induced & $\begin{array}{c}\text {-Fold } \\
\text { induction }\end{array}$ & Basal Induced & $\begin{array}{c}\text {-Fold } \\
\text { induction }\end{array}$
\end{tabular}

$\begin{array}{lrrrrrr}\text { CYP3A4 } & & & & & & \\ \text { Donor 1 } & 98 & 257 & 2.62 & 39 & 84 & 2.14 \\ \text { Donor 2 } & 112 & 319 & 2.85 & 48 & 107 & 2.23 \\ \text { Donor 3 } & 90 & 239 & 2.66 & 32 & 62 & 1.96 \\ & & & & & & \\ \text { CYP2C9 } & & & & & & \\ \text { Donor 1 } & 91 & 181 & 1.99 & 29 & 43 & 1.52 \\ \text { Donor 2 } & 103 & 425 & 4.13 & 34 & 106 & 3.13 \\ \text { Donor 3 } & 48 & 82 & 1.71 & 9 & 14 & 1.51\end{array}$

The activities of PHH are reflected by the corresponding $\mathrm{MH}$ cells, suggesting the possibility to predict individual CYP activities using $\mathrm{MH}$ cells.

well as the difficult, time consuming and expensive generation process. Another innovative approach is the direct conversion of fibroblasts to hepatocyte-like cells by genetic modifications. ${ }^{16,17}$ Yet, this procedure has until now not been established for human tissue and possible interferences of the genetic modifications with individual cell functions are not predictable.

As a possible solution we propose $\mathrm{MH}$ cells. $\mathrm{MH}$ cells can be generated from peripheral monocytes efficiently in a period of 10 days and show several specific enzyme activities that are also present in PHHs. These enzyme activities are found with low variability and high reproducibility, suggesting a robust generation protocol. Furthermore, MH cells show urea secretion dependent on ammonium chloride exposure and menadione-dependent secretion of coagulation factor VII. Thus, there seem to be several hepatocyte-like features in this cell model. $\mathrm{MH}$ cells possess inducible CYP450 enzymes and their CYP functions are preserved for at least 4 weeks in culture.

Using luminescent assays, we could show that there are relevant activities of the CYP enzymes 1A2, 2C9 and 3A4 in $\mathrm{MH}$ cells, which are inducible as in PHHs. Moreover, protein expression of CYP 3A4 and 2E1 was confirmed by western blotting showing $50-60 \%$ of CYP3A4 and $2 \mathrm{E} 1$ protein expression in $\mathrm{MH}$ cells compared with PHHs. As this is the first article that describes the properties of $\mathrm{MH}$ cells and the luminescent technique is only applicable for selected CYP enzymes not all of the important members of the CYP450 family were investigated. More data are needed for CYP2C19, $2 \mathrm{~A} 6$ and 2B6. Studies employing metabolite analyses to measure activities of the other important CYP enzymes are under way. CYP2C19 is of special interest, because it is inducible and genetic polymorphisms of this enzyme are well characterized. One of the next steps will be the analysis of $S$-mephenytoin metabolism and its induction in donors with known 2C19 genotype. Our data support hepatocytelike functional CYP induction in MH cells by ligands of the PXR, of the aryl-hydrocarbon receptor, of the glucocorticoid receptor and of the constitutive androstendione receptor. The latter is lacking in some hepatoma cell models, ${ }^{21}$ but its presence is crucial for the predictive value of induction studies. Evidence for the presence of nuclear orphan receptors is also supported by the microarray data, a more detailed study of nuclear orphan receptors in $\mathrm{MH}$ cells is planned.

Furthermore, MH cells exhibit toxicity of APAP, which has to be metabolized by CYP 2E1 to exert toxic effects. In MH cells, APAP induces depletion of thiol groups as a surrogate marker for oxidative stress, which is in accordance with the pathophysiology of APAP hepatotoxicity. These data highlight the capacity of the cells for metabolic activation of drugs via the CYP450 enzyme system. Our data show the clinically important interaction of ethanol and APAP, a prime example for modulation of drug toxicity. $\mathrm{MH}$ cells prove to be a model for this dangerous interaction.

Moreover, long-term stability could make $\mathrm{MH}$ cells a unique model to investigate individual metabolic capacities and perhaps even a tool for metabolic phenotyping, for example, a diagnostic marker to prevent drug toxicity. As there are no data available on another in vitro model that maintains hepatocyte functions for $>2$ weeks without complicated culture methods, this unrivalled feature of $\mathrm{MH}$ cells could enable replacement of animal testing for longterm hepatic toxicity and drug interactions.

Interestingly, $\mathrm{MH}$ cells seem to reflect gender characteristics of their donors. We found that there are gender-specific differences in CYP450 enzyme activities, which translate into different in vitro toxicities, as shown for diclofenac. Moreover, the CYP metabolism of these cells allows experimental set-ups that can uncover potentially dangerous drug interactions in the preclinical development as we could show for the prime example of ethanol and APAP and the interaction of rifampicin and diclofenac. The enhanced toxicity of APAP after prior exposure to ethanol is a clinical setting that is reflected by the MH cells, underlining the possibilities of this model. MH cells can be cryoconserved and this enables the establishment of a cell bank, which can be used together with clinical data to screen substances for toxicity and metabolism in silico in a 'population' of donors. Thus, hepatocyte-like $\mathrm{MH}$ cells could be an enabling tool in drug discovery and development.

In a small group of patients, we found that monocytederived cells reflect donor-specific CYP activities and induction. Similar results were found by analysis of gene expression pattern focusing on genes involved in metabolism and toxicity. Thus, it is conceivable, that cells from an 
a

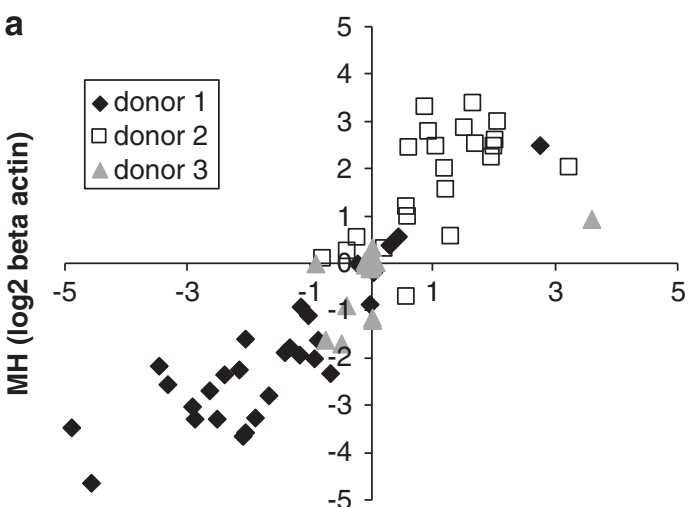

PHH (log2 beta actin) b

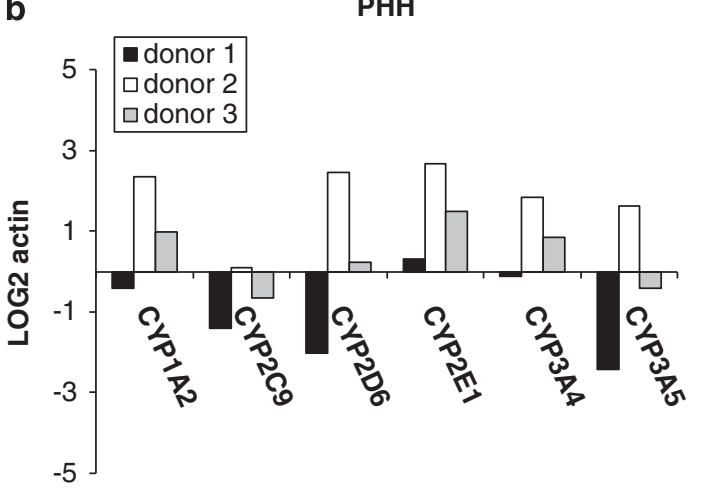

C

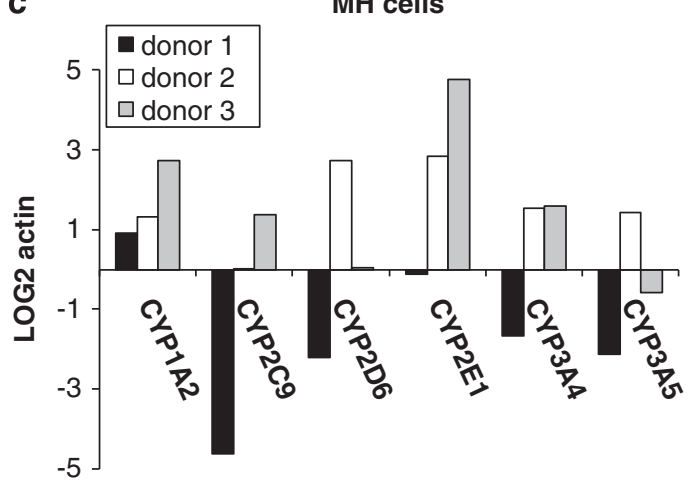

Figure 5 (a) Correlation of expression levels of transcription factors between PHH and MH cells from three individual donors. Data were normalized to beta-actin mRNA and expression levels are presented as LOG2. Correlation coefficients were 0.91 for donor $1,0.81$ for donor 2 and 0.51 for donor 3 , respectively. (b) Basal CYP-mRNA expression in PHH derived from three individual donors. (c) CYP mRNA expression of MH cells derived from the same three donors. MH cells show similar expression patterns of basal CYP-mRNA as the corresponding PHH.

individual patient could be applied in clinical medicine to screen in advance a potentially toxic drug regimen for a given patient. Most interesting, the best correlations were found in expression of transcription factors. This could point toward an induction of hepatocyte gene expression in monocytes by the applied culture protocol. Yet, this has to be validated further.

In summary, we introduce a new model for in vitro toxicology and CYP metabolism research, which has several advantages: the system is stable and reproducible and provides a good approximation to PHHs. In contrast to stem cell approaches, cell yield is high and the generation process is cost effective and quick. The cells can be cryoconserved to enable the establishment of a cell bank and the further research on cells from a specific donor. CYP activities show long-term-stability for at least 28 days, which can be used for chronic toxicity experiments. Moreover, the ability of $\mathrm{MH}$ cells to model drug interactions in vitro provides novel possibilities for investigation of drug interaction together with long-term experiments. The differences between individuals and genders that are supported by our findings in combination with cryoconservation enables research on individuals as well as a 'population-based' approach using $\mathrm{MH}$ cells from donors with different gender and co-medications.
Supplementary Information accompanies the paper on the Laboratory Investigation website (http://www.laboratoryinvestigation.org)

\section{ACKNOWLEDGEMENT}

This work was funded by FöFoLe Grant 563/546 and 14/2008 LudwigsMaximilians-University, Munich, Germany and the Gesellschaft für Gastroenterologie in Bayern eV., Sauerlach, Germany. The study was supported by HTCR, a non-profit foundation under German civil law, which facilitates research with human tissue by providing an ethical and legal framework for prospective sample collection.

\section{AUTHOR CONTRIBUTIONS}

Andreas Benesic wrote the paper, planned and performed experiments, analyzed data, performed statistics and obtained funding. Nora Rahm performed experiments and obtained funding. Samuel Ernst performed experiments. Alexander L Gerbes critically revised the manuscript, planned experiments, supervised the study and obtained funding.

\section{DISCLOSURE/CONFLICT OF INTEREST}

The authors disclose potential financial conflicts, since a patent application has been filed for the generation of $\mathrm{MH}$ cells as well as all possible applications.

1. Pelkonen $\mathrm{O}$, Turpeinen $\mathrm{M}$, Hakkola J, et al. Inhibition and induction of human cytochrome P450 enzymes: current status. Arch Toxicol 2008;82:667-715.

2. Watkins RE, Wisely GB, Moore LB, et al. The human nuclear xenobiotic receptor PXR: structural determinants of directed promiscuity. Science 2001;292:2329-2333. 
3. Nebert DW, Russell DW. Clinical importance of the cytochromes P450. Lancet 2002;360:1155-1162.

4. Zanger UM, Klein K, Richter T, et al. Impact of genetic polymorphism in relation to other factors on expression and function of human drugmetabolizing p450s. Toxicol Mech Methods 2005;15:121-124.

5. Klein K, Winter S, Turpeinen $\mathrm{M}$, et al. Pathway-targeted pharmacogenomics of CYP1A2 in human liver. Front Pharmacol 2010;1:129.

6. Schirmer M, Rosenberger A, Klein K, et al. Sex-dependent genetic markers of CYP3A4 expression and activity in human liver microsomes. Pharmacogenomics 2007;8:443-453.

7. Scandlyn MJ, Stuart EC, Rosengren RJ. Sex-specific differences in CYP450 isoforms in humans. Expert Opin Drug Metab Toxicol 2008;4:413-424.

8. Zanger UM, Turpeinen M, Klein $\mathrm{K}$, et al. Functional pharmacogenetics/ genomics of human cytochromes P450 involved in drug biotransformation. Anal Bioanal Chem 2008;392:1093-1108.

9. Burk O, Schwab M. The limited impact of CYP3A5 genotype for the pharmacokinetics of CYP3A substrates. Eur J Clin Pharmacol 2007;63: 1097-1098.

10. Paré G, Mehta SR, Yusuf S, et al. Effects of CYP2C19 genotype on outcomes of clopidogrel treatment. N Engl J Med 2010;363:1704

11. Yang $X$, Zhang B, Molony $C$, et al. Systematic genetic and genomic analysis of cytochrome P450 enzyme activities in human liver. Genome Res 2010;20:1020-1036.

12. Parkinson A, Mudra DR, Johnson C, et al. The effects of gender, age, ethnicity, and liver cirrhosis on cytochrome P450 enzyme activity in human liver microsomes and inducibility in cultured human hepatocytes. Toxicol Appl Pharmacol 2004;199:193-209.

13. Guguen-Guillouzo C, Guillouzo A. General review on in vitro hepatocyte models and their applications. Methods Mol Biol 2010;640:1-40.

14. Hewitt NJ, Hewitt P. Phase I and II enzyme characterization of two sources of HepG2 cell lines. Xenobiotica 2004:34:243-256.

15. Mavri-Damelin D, Eaton $\mathrm{S}$, Damelin $\mathrm{LH}$, et al. Ornithine transcarbamylase and arginase I deficiency are responsible for diminished urea cycle function in the human hepatoblastoma cell line HepG2. Int J Biochem Cell Biol 2007;39:555-564.

16. Sekiya S, Suzuki A. Direct conversion of mouse fibroblasts to hepatocyte-like cells by defined factors. Nature 2011;475:390-393.

17. Huang $\mathrm{P}, \mathrm{He} Z$, Ji S, et al. Induction of functional hepatocyte-like cells from mouse fibroblasts by defined factors. Nature 2011;475:386-389.

18. Yan L, Han Y, Wang J, et al. Peripheral blood monocytes from patient with HBV related decompensated liver cirrhosis can differentiate into functional hepatocytes. Am J Hematol 2007;82:949-954.

19. Dambach DM, Watson LM, Gray KR, et al. Role of CCR2 in macrophage migration into the liver during acetaminophen-induced hepatotoxicity in the mouse. Hepatology 2002;35:1093-1103.
20. Rothenberg EV. Negotiation of the $T$ lineage fate decision by transcription-factor interplay and microenvironmental signals. Immunity 2007:26:690-702.

21. uhnke $M$, Ungefroren $H$, Nussler $A$, et al. Differentiation of in vitromodified human peripheral blood monocytes into hepatocyte-like and pancreatic islet-like cells. Gastroenterology 2005;128:1774-1786.

22. Riquelme $\mathrm{P}$, Wundt J, Hutchinson JA, et al. A refined characterisation of the NeoHepatocyte phenotype necessitates a reappraisal of the transdifferentiation hypothesis. Differentiation 2009;77:263 .

23. Ehnert S, Seeliger $\mathrm{C}$, Vester $\mathrm{H}$, et al. Autologous serum improves yield and metabolic capacity of monocyte-derived hepatocyte-like cells: possible implication for cell transplantation. Cell Transplant 2011; 20:1465-1477.

24. Thasler WE, Weiss TS, Schillhorn K, et al. Charitable state-controlled foundation human tissue and cell research: ethic and legal aspects in the supply of surgically removed human tissue for research in the academic and commercial sector in Germany. Cell Tissue Banking 2003:4:49-56.

25. De Toni EN, Kuntzen C, Gerbes AL, et al. P60-c-src suppresses apoptosis through inhibition of caspase 8 activation in hepatoma cells, but not in primary hepatocytes. J Hepatol 2007;46:682-691.

26. Schumann G, Klauke R. New IFCC reference procedures for the determination of catalytic activity concentrations of five enzymes in serum: preliminary upper reference limits obtained in hospitalized subjects. Clin Chim Acta 2003;327:69-79.

27. Benesic A, Schwerdt G, Freudinger $R$, et al. Chloroacetaldehyde as a sulfhydryl reagent: the role of critical thiol groups in ifosfamide nephropathy. Kidney Blood Press Res 2006;29:280-293.

28. Cali JJ, Ma D, Sobol M, et al. Luminogenic cytochrome P450 assays. Expert Opin Drug Metab Toxicol 2006;2:629-645.

29. Hariparsad N, Carr BA, Evers R, et al. Comparison of immortalized Fa2N4 cells and human hepatocytes as in vitro models for cytochrome P450 induction. Drug Metab Dispos 2008;36:1046-1055.

30. Hinson JA, Roberts DW, James LP. Mechanisms of acetaminopheninduced liver necrosis. Handb Exp Pharmacol 2010;196:369-405.

31. Tang $W$. The metabolism of diclofenac-enzymology and toxicology perspectives. Curr Drug Metab 2003;4:319-329.

32. Yan Z, Li J, Huebert $\mathrm{N}$, et al. Detection of a novel reactive metabolite of diclofenac: evidence for CYP2C9-mediated bioactivation via arene oxides. Drug Metab Dispos 2005;33:706-713.

33. Colmenero J, Bataller R, Sancho-Bru P, et al. Hepatic expression of candidate genes in patients with alcoholic hepatitis: correlation with disease severity. Gastroenterology 2007;132:687-697.

34. Jaeschke $\mathrm{H}$, Gores $\mathrm{GJ}$, Cederbaum Al, et al. Mechanisms of hepatotoxicity. Toxicol Sci 2002;65:166-176. 